\title{
Sudden Cardiac Arrest Caused by a Ball Impact to the Chest Wall: Commotio Cordis
}

\author{
Anmar Mandourah
}

\begin{abstract}
A sudden cardiac death caused by a blunt forceful trauma in the chest wall region is called commotio cordis. It is considered to be the second leading cause of sudden death in a young athlete after hypertrophic obstructive cardiomyopathy. The number of reported cases is increasing by the year and that corresponds to the increased awareness of the condition. Commotio cordis by definition is blunt trauma to the chest wall in the area of the cardiac silhouette causing a ventricular fibrillation. The actual mechanism of the induction of the ventricular fibrillation is complicated but can be simplified as a forced depolarization in the impact area caused by a disturbance of the $\mathrm{K}^{\mathrm{ATP}}$ channel, which, if happened in a specific time in the cardiac cycle specifically within 20 - $10 \mathrm{~ms}$ before the $\mathrm{T}$ wave peak, will lead to a ventricular fibrillation. Here, we discuss a 25 -year-old man that got hit with a tennis ball during a match and was luckily revived with the aid of an automated external defibrillator (AED).
\end{abstract}

Keywords: Commotio cordis; Tri-fascicular block; Dual-chamber ICD

\section{Introduction}

Commotio cordis is a quite uncommon condition that can affect young individuals aged $<25$ years old [1]. Given the nature of the condition, the numbers of survivors are quite few. Especially considering recreational sports, where the availability of emergency medical equipment such as an automated external defibrillator (AED) could be limited. As the condition is quite a rare occurrence, a sudden impact on the chest wall in a young individual occurring in a specific period of the cardiac cycle just before the peak of the T wave about $20-10 \mathrm{~ms}$ which accounts for only $1 \%$ of the whole cycle [1], and in addition to the lower survival rate, we recon our case as an interesting case to study given that the patient survived the cardiac arrest.

Manuscript submitted October 19, 2017, accepted November 10, 2017

Department of Cardiology, Skane University Hospital, Malmo, Sweden. Email: ajmandourah@gmail.com

doi: https://doi.org/10.14740/jmc2941w

\section{Case Report}

A 25-year-old man, known with an intellectual disability otherwise previously healthy, was playing tennis while he got struck forcefully by the tennis ball on his chest wall and fell on his back. The patient got up on his feet and continued playing for approximately $2 \mathrm{~min}$ and then suddenly fell on the ground and became cyanotic. His dad who was there stated that the patient was completely lifeless. Three doctors, which happened to be in the same tennis court, began cardiopulmonary resuscitation (CPR) and the AED suggested a defibrillation. After two cycles of CPR with two defibrillations, a return of spontaneous circulation (ROSC) was obtained. An atrial fibrillation was noted in the ambulance. Upon arrival to the hospital, another ECG was ordered which showed an atrial flutter with 2:1 3:1 block, 150 beats per minute and a right bundle branch block (RBBB). Cardioversion was performed and sinus rhythm was obtained. The control ECG after the cardioversion showed a sinus rhythm. The heart rate was about 84 beats per minute. Long PQ interval as with a first-degree AV block, RBBB and a left anterior hemiblock (LAHB), were found. They form combined - a so called tri-fascicular block (Fig. 1).

Laboratory studies revealed a slight increase in creatinine clearance, potassium and sodium which have normalized after around $4 \mathrm{~h}$. C-reactive protein (CRP) and leukocytes were normal $\mathrm{Hb}$ was normal. Troponin- $\mathrm{T}$ was 264 and decreased to 170 after around $4 \mathrm{~h}$. There was a slight increase in liver parameters primarily ASAT and ALAT probably secondary to the cardiac arrest.

Coronary computed tomography angiography (CTA) showed no signs of neither stenosis in the coronary arteries nor ateromatosis. CT of thorax with contrast excluded pulmonary embolism. A little parenchymal change in both lungs was noted which can be due to lung contusion secondary to the impact.

Echocardiography showed a normal sized left ventricle, and apicolateral hypokinesia. Ejection fraction was around $50 \%$. Size of right ventricle was normal with a normal systolic function. Size of left atrium was normal. The inferior vena cava was of a normal width. Valves were normal. Doppler showed no pulmonary hypertension.

Cardiac magnetic resonance imaging (MRI) showed a normal sized left ventricle, and no hypertrophy was noted. There were no signs of infarction or fibrosis. A myocarditis was suspected in the inferolateral region, but it was not clear if it was old or new. Overall the image did not fill the criteria for arrhythmogenic right ventricular dysplasia (ARVD). 


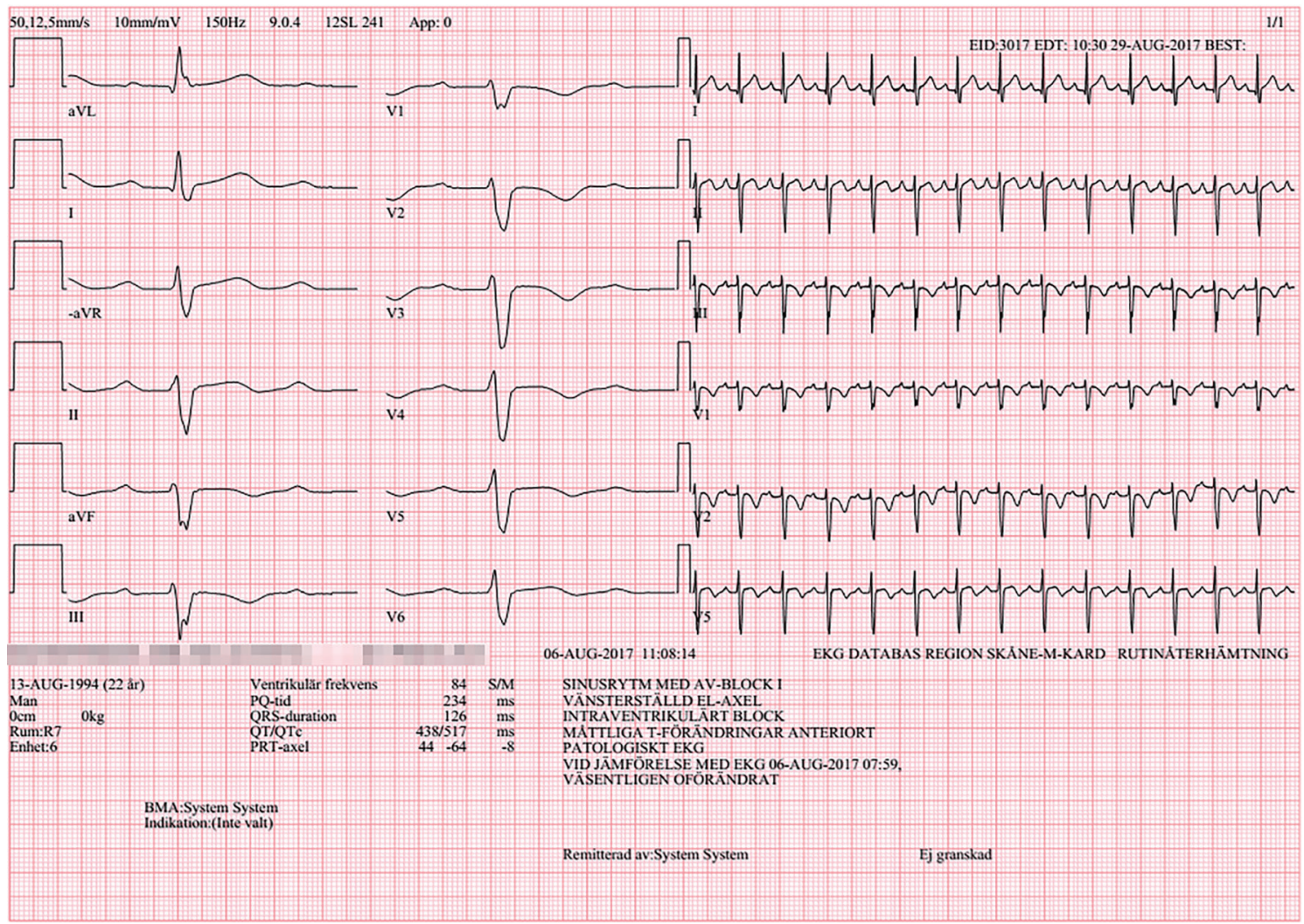

Figure 1. Patient's electrocardiogram after cardioversion. This is the ECG taken after the successful cardioversion. It shows a right bundle branch block, a left anterior hemiblock and a first-degree AV block.

Under the observation of the telemetry in around 2 weeks in the ward, no tachyarrhythmias or bradyarrhythmias were noted. The case was lifted up in an arrhythmia conference for discussion where a decision of implanting a secondary prophylaxis dual-chamber implantable cardioverter defibrillator (ICD) has been made given the possibility of bradyarrhythmiainduced tachyarrythmia keeping in mind the patient's tri-fasicular block which was unsure if it was a new or an old finding as well as the fact that a recurrence of tachyarrhythmia cannot also be excluded.

A 1-month control of the ICD showed pacing $<1 \%$. No tachyarrhythmia or bradyarrhythmia was recorded. A control echocardiography showed a normal EF. The size of left/right ventricles was normal. Valves were normal.

\section{Discussion}

Commotio cordis is a diagnosis of exclusion, typically more common in young individuals. A number of criteria must be met for a cardiac arrhythmia to be triggered in such cases. These include the size of the object, the speed/force of the impact, the impacted area of the chest wall, and the timing of the impact in the cardiac cycle. The mechanical force of the impact triggers the $\mathrm{K}^{\mathrm{ATP}}$ channel which is stretch-sensitive leading to depolarization of the myocytes in the impacted area, a ventricular fibrillation (VF) trigger [1-5]. Although the L-type calcium channels also could be affected, they do not play a big role in the mechanism of commotio cordis [4]. Despite that, a substrate is needed to trigger a VF as the triggered premature ventricular depolarization is not a sufficient cause of VF, it is strongly believed that the activation of the stretch-sensitive channels leads to an increase in the dispersion of repolarization which when added to the already vulnerable period near the peak of the T wave, can be considered as sufficient [6]. A non-synchronized cardioversion should be performed as soon as possible to avoid organ damage.

Ideally a well-timed impact to the chest wall will cause a VF [1-7]. In our case though, the patient got up on his feet and continued playing for $2 \mathrm{~min}$. This could be explained by a ventricular tachycardia which progressed to a fibrillation where the AED suggests a defibrillation. Such phenomenon has been reported before [5]. Due to the lack of an old ECG to compare with, we cannot be certain that the patient's rhythm, RBBB, first-degree AV block and LAHB, is new or old. The decreased ejection fraction and the apicolateral hypokinesia are not so uncommon in such presentation [6], although it can also be explained by the suspected myocarditis finding in the cardiac MRI. The absence of pathological findings in CT angiography, thorax and MRI - other than the suspected myocarditis - on the other hand, supports the diagnosis.

The lack of evidence in patients with commotio cordis is huge. There is no evidence that the affected individuals are in an increased risk of cardiac arrhythmias. In the same manners, 
there is no evidence that ICD is indicated in such patients [1]. Our patient got a dual-chamber ICD as secondary prophylaxis. Our thinking was about the possibility of bradyarrhythmiainduced ventricular tachyarrhythmias, keeping in mind the trifasicular block, in addition to the uncertainty of a possibility of recurrent ventricular arrhythmias in the future.

In the 1-month control of the patient's ICD, a less than $1 \%$ of pacing is registered. No arrhythmias were registered. A control echocardiography showed a normal EF. The size of left/right ventricles was normal. Valves are normal.

The number of reported cases of commotio cordis is increasing, which reflects the raised awareness of such a case. Additionally, the numbers of survived reported cases are also rising, which can relate to the increased awareness of the importance of bystander basic life support and the usage of AED [7].

\section{Reference}

1. Maron BJ, Estes NA, 3rd. Commotio cordis. N Engl J
Med. 2010;362(10):917-927.

2. Kohl P, Nesbitt AD, Cooper PJ, Lei M. Sudden cardiac death by Commotio cordis: role of mechano-electric feedback. Cardiovasc Res. 2001;50(2):280-289.

3. Menezes RG, Fatima H, Hussain SA, Ahmed S, Singh PK, Kharoshah MA, Madadin M, et al. Commotio cordis: A review. Med Sci Law. 2017;57(3):146-151.

4. Madias C, Garlitski AC, Kalin J, Link MS. L-type calcium channels do not play a critical role in chest blow induced ventricular fibrillation: Commotio Cordis. Cardiol Res Pract. 2016;2016:5191683.

5. Wolbrom DH, Rahman A, Tschabrunn CM. Mechanisms and Clinical Management of Ventricular Arrhythmias following Blunt Chest Trauma. Cardiol Res Pract. 2016;2016:7270247.

6. Madias C, Maron BJ, Weinstock J, Estes NA, 3rd, Link MS. Commotio cordis - sudden cardiac death with chest wall impact. J Cardiovasc Electrophysiol. 2007;18(1):115122.

7. Link MS. Pathophysiology, prevention, and treatment of commotio cordis. Curr Cardiol Rep. 2014;16(6):495. 